Rev. Elev. Méd. vét. Pays trop., 1974, 27 (4) : 479-485

\title{
Influence du mode d'exploitation sur la productivité des pâturages naturels de Madagascar. Conséquences
}

\author{
par P. GRANIER $\left(^{*}\right)$, Y. CABANIS, A. BIGOT
}

\begin{abstract}
RESUME
Le mode d'exploitation (dates et nombre de coupes) influe sur la productivité globale et saisonnière différemment selon le type de pâturage (zone humide ou sèche) et le climat.

L'expérimentation a permis de préciser les relations entre le climat, les sols et la végétation et de préconiser un mode d'exploitation en fonction à la fois de la spéculation envisagée et de la zone intéressée.
\end{abstract}

\section{ETUDE EXPERIMENTALE}

$\mathrm{Au}$ cours d'une première phase de travaux, la productivité théorique de l'herbe $(3,4)$ a été étudiée pour chaque type de pâturage dans les deux Centres de Recherches de l'I.E.M.V.T. à Madagascar dont les caractéristiques écologiques sont les suivantes :

\section{C.R.Z.F. Kianjasoa}

- Domaine du Centre (Moyen-Ouest).

- $1600 \mathrm{~mm}$ de pluies, 120 jours de pluies/an.

- 27 p. 100 de bas-fonds humides toute l'année, sols ferralitiques.

\section{C.R.Z.F. Miadana}

- Domaine de l'Ouest.

- $1200 \mathrm{~mm}$ de pluies, 80 jours de pluies/an.

- 4 p. 100 de bas-fonds se desséchant en fin de saison sèche. Sols sablonneux, sédimentaires.

Ces premiers travaux ont permis de donner, avec la productivité théorique du pâturage, la

(I.E.M.V.T., Région de Recherches vétérinaires et zootechniques de Madagascar, B.P. 4, Tananarive.

(*) Adresse actuelle: Laboratoire de l'Elevage, B.P. 485, Niamey (Niger). charge à l'hectare, le nombre de coupes ou de passages, la durée des temps de repos selon la saison et les divers types de pâturages.

La présente note permet de déterminer les variations de la productivité selon le mode d'exploitation. Reprenant les mêmes parcelles témoins qui avaient servi à calculer l'évolution des rendements de chaque association végétale représentative d'un type de pâturage, on a fait varier le nombre de coupes. En se basant sur une étude du comportement du bétail (2) qui montrait que celui-ci ne pâturait pratiquement plus sur les terres hautes en saison sèche, mais surpâturait les bas-fonds, on a étudié ce que devenait la productivité en raccourcissant les temps de repos en saison des pluies sur les terres hautes, et en saison sèche sur les terres basses pour se rapprocher des conditions naturelles.

Nous avons distingué, pour les bas-fonds, un mode d'exploitation continu, qui consiste à couper l'herbe chaque fois qu'elle atteint la hauteur de pâture $(25 \mathrm{~cm})$, d'un mode d'exploitation rationné dans lequel le pâturage est sousexploité en saison des pluies afin de voir s'il y avait une augmentation concomitante de la productivité en saison sèche. 


\section{RESULTATS}

\section{A. PATURAgeS DE SAISON DES PLUIES}

Les études avaient pour but de montrer si le mode d'exploitation avait une influence sur le rendement global annuel et si les variations permettaient d'apporter une contribution à la connaissance des milieux (Ouest et Moyen-Ouest).

\section{Moyen-Ouest (Kianjasoa) (Graph. I)}

Au cours des années 1966-1967, des carrés de $4 \mathrm{~m}^{2}$ ayant un coin commun, situés sur une bande de végétation homogène étaient fauchés tous les 10 jours afin de permettre l'établissement de courbes de croissance de l'herbe sous l'influence de divers traitements.

En 1968 et 1969, le protocole prévoyait que la totalité de la bande de végétation homogène serait fauchée lorsque la hauteur moyenne de la strate herbacée atteindrait $35 \mathrm{~cm}$ de hauteur et qu'à partir de cette coupe, des carrés de $4 \mathrm{~m}^{2}$ (deuxième cycle) seraient fauchés tous les 15 jours environ (comme l'on avait procédé dans les expérimentations précédentes).

\section{Résultats}

- Première coupe: le 19 février.

- Rendement en $t / h a /$ vert : 7,5 .

- Teneur en matière sèche: 27 p. 100 .

- Rendement en t/ha/M.S. : 2,0.

- Productivité du deuxième cycle.

\begin{tabular}{rccc}
\hline $\begin{array}{c}\text { Dates } \\
\text { des } \\
\text { coupes }\end{array}$ & $\begin{array}{c}\text { Rendement } \\
\text { en } \\
\text { t/ha/vert }\end{array}$ & $\begin{array}{c}\text { Pour- } \\
\text { centage } \\
\text { M.S. }\end{array}$ & $\begin{array}{c}\text { Rendement } \\
\text { en } \\
\text { t/ha/M.S. }\end{array}$ \\
\hline 5 mars & 0,625 & 20 & 0,125 \\
20 mars & 1,52 & 22 & 0,33 \\
4 avril & 2,25 & 25 & 0,56 \\
19 avril & 3,37 & 28 & 0,94 \\
4 mai & 3,8 & 29 & 1,10 \\
20 mai & 4,5 & 29 & 1,3 \\
3 juin & 3,5 & 35 & 1,25 \\
\hline
\end{tabular}

A partir de cette date, le pâturage se dessèche, les rendements diminuent du fait de la chute des feuilles et des fragments de chaumes : le développement du deuxième cycle est terminé, le rendement en M.S. se stabilise ensuite aux environs de 1,2 t/ha.
La productivité maximale des regains est donc de $1,3 \mathrm{t} / \mathrm{ha} / \mathrm{M} . \mathrm{S}$. le 20 mai.

La productivité cumulée de la parcelle est de :

$$
2,0+1,3=3,3 \mathrm{t} / \mathrm{ha} / \text { M.S. }
$$

Or, la coupe unique d'une bande témoin donne à la même date un rendement pratiquement identique de 3,4 t/ha/M.S.

\section{Conclusions}

a) Le nombre de coupes ne modifie pas la productivité annuelle.

b) La multiplication des coupes n'influe pas sur les dates d'exploitation, les rendements maximaux des regains se situent à peu près à la même date que ceux de la coupe unique.

\section{Ouest (Miadana) (Graph. II)}

Avec la même méthode, on obtient les rendements suivants :

- Coupe unique

(t/ha/M.S.)

21 avril $=13,5$ à 28 p. 100 M.S. $=3,78$

6 mai $=17$ à 30 p. 100 M.S. $=5,1$

- Productivité cumulée de deux coupes

21 janv. $=6,6$ à 20 p. 100 M.S. $=1,32$

21 avril $=13,3$ à 28 p. 100 M.S. $=3,72$

5,04

31 janv. $=7$ à 22 p. 100 M.S. $=1,54$

6 mai $=15$ à 30 p. 100 M.S. $=4,50$

6,04

10 févr. $=7,3$ à 24 p. 100 M.S. $=1,75$

6 mai $=11$ à 30 p. 100 M.S. $=3,3$

5,05

Nous pouvons conclure que dans l'Ouest:

a) La productivité cumulée de deux coupes successives peut être supérieure à la productivité d'une seule coupe.

b) Le temps de repos en saison des pluies est d'environ 2 mois et la productivité des regains est maximale si le temps de repos est d'environ 3 mois.

Il est donc nécessaire, pour tenir compte de la date d'arrêt des pluies qui conditionne la croissance des regains que la première exploitation soit effectuée à la fin du mois de janvier. 
Graph. I.

EXPLOITATION DU PATURAGE NATUREL MOYEN_ OUEST

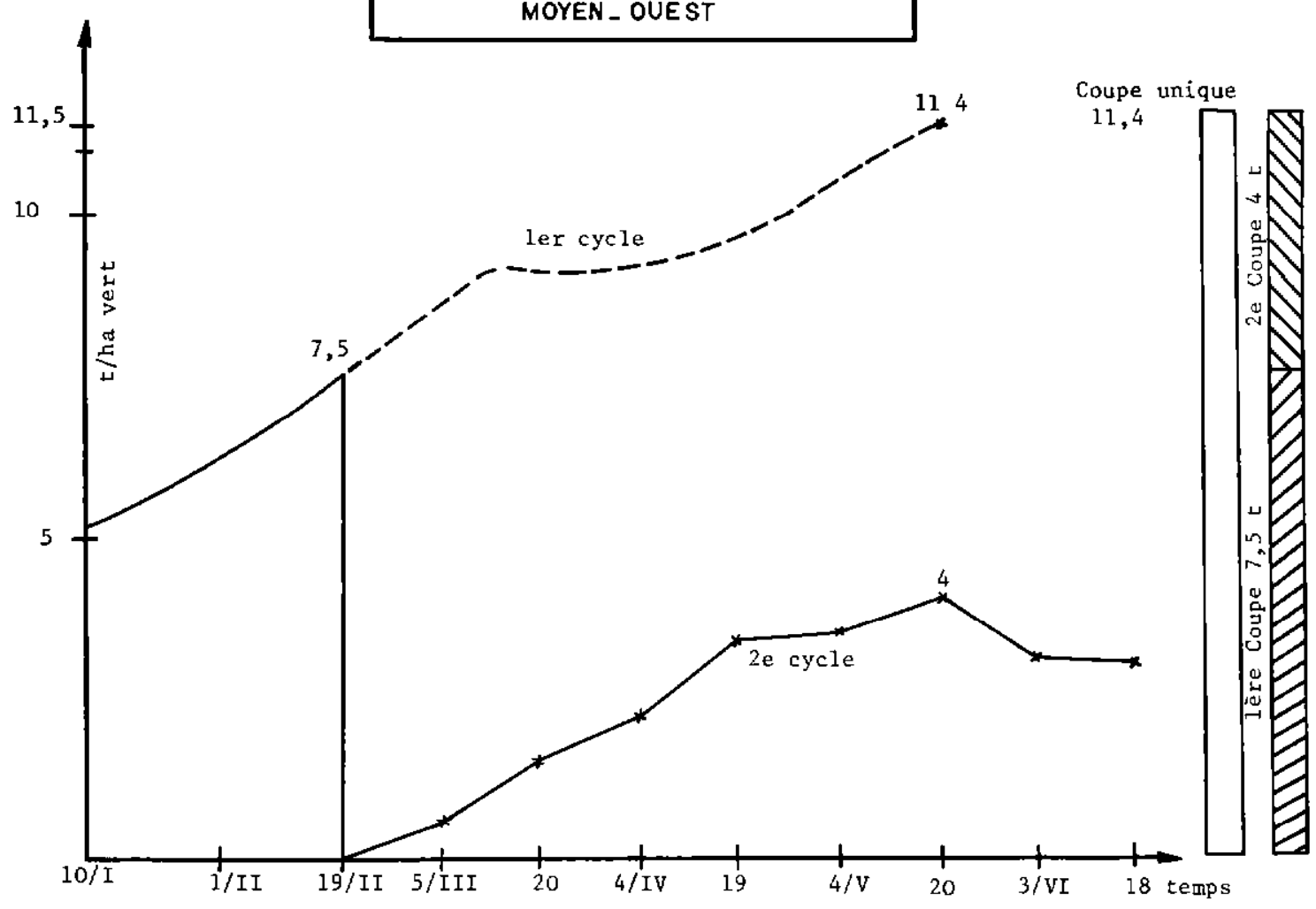

Graph. II.

EXPLOITATION DU PATURAGE NATUREL OUEST (MIADANA)

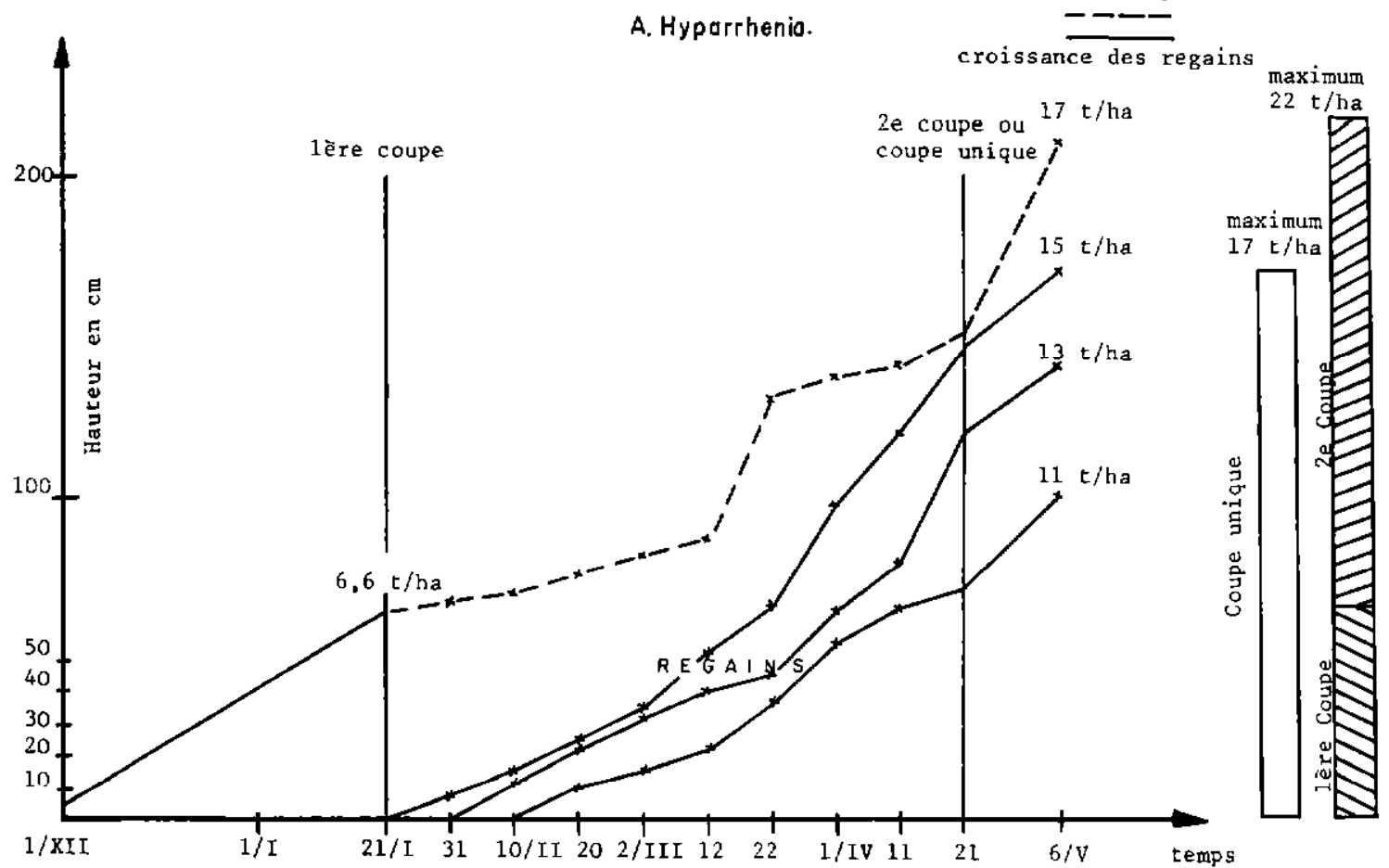




\section{B. PATURAGES DE SAISON SECHE}

\section{Moyen-Ouest (Kianjasoa)}

Les tableaux I et II donnent les variations de la productivité des pâturages des mêmes associations végétales de bas-fond exploités soit en continu, soit en rationné comme il a été défini ci-dessus.

\section{On remarque :}

a) que dans le cas d'une exploitation rationnée, la productivité annuelle est toujours inférieure à celle obtenue en exploitation continue. En " continu ", on fait 2 ou 3 coupes en saison des pluies et en "rationné " une seule, au moment où la production de matière sèche par jour est la plus élevée. Il y a inévitablement une perte que l'on ne peut compenser sur un bilan annuel;

b) Ia productivité en saison sèche est toujours plus élevée en exploitation rationnée (3 coupes au lieu de 2). L'allongement du temps de repos en saison des pluies permet d'effectuer une coupe supplémentaire en pleine saison sèche et c'est là l'intérêt essentiel de ce mode d'exploitation, la charge du bas-fond en saison sèche étant le facteur limitant la productivité de l'élevage en général;

c) l'accroissement de la productivité est nettement plus élevé pour la prairie humide que pour les colluvions qui se comportent comme un pâturage de terres hautes, le bilan hydrique des sols étant déficitaire en fin de saison sèche.

\section{Ouest (Miadana)}

Une répétition de cette expérimentation sur les bas-fonds du CRZF de Miadana donne des résultats très différents parce que l'approvisionnement en eau de ces terres n'est pas suffisant pour maintenir une croissance de l'herbe continue. La végétation, par ailleurs, est composée non par des graminées basses comme dans le Moyen-Ouest (Panicum, Leersia, Cynodon) mais par de grandes graminées (Hyparrhenia, Echinochloa, Ischaemum) qui sont déprimées par des coupes répétées.

TABLEAU N* I.-Productivitës comparëes moyennes en UF par ha(Moyen-Ouest)

\begin{tabular}{|c|c|c|c|c|c|c|c|}
\hline & & \multicolumn{3}{|c|}{ Rendement moyen en $t$ /ha M.S. } & \multicolumn{3}{|c|}{$\begin{array}{l}\text { Rendement moyen en U.F. } \\
\text { (dêduction faite des refus) }\end{array}$} \\
\hline & & Annue 1 & $\begin{array}{l}\text { Salson } \\
\text { des pluies }\end{array}$ & $\begin{array}{l}\text { Saison } \\
\text { sèche }\end{array}$ & Annuel & $\begin{array}{l}\text { Saison } \\
\text { des pluies }\end{array}$ & $\begin{array}{l}\text { Saison } \\
\text { sèche }\end{array}$ \\
\hline \multirow{2}{*}{$\begin{array}{l}\text { Prairze } \\
\text { humide }\end{array}$} & $\begin{array}{l}\text { Pâturage } \\
\text { continu }\end{array}$ & 11,6 & 7,6 & 4 & 4065 & 2930 & 1135 \\
\hline & $\begin{array}{l}\text { Pâturage } \\
\text { rationne }\end{array}$ & 10,2 & 3 & 7,2 & 3270 & 1170 & 2100 \\
\hline \multirow{2}{*}{ Colluvions } & $\begin{array}{l}\text { Pâturage } \\
\text { continu }\end{array}$ & 6,6 & 5,8 & 0,8 & 2155 & 1920 & 235 \\
\hline & $\begin{array}{l}\text { Pâturage } \\
\text { rationné }\end{array}$ & 4,5 & 3,0 & 1,5 & 1610 & 1170 & 440 \\
\hline
\end{tabular}

TABLEAU $\mathbb{N}^{\circ}$ II,-Productivité des bas fonds (par ha gëographique) (Moyen-Ouest).

\begin{tabular}{|c|c|c|c|c|c|c|c|}
\hline $\begin{array}{l}\text { Mode } \\
\text { d'exploitation }\end{array}$ & $\begin{array}{l}\text { Type de } \\
\text { pâturages }\end{array}$ & \multicolumn{3}{|c|}{$\begin{array}{c}\text { Productivité annue1le } \\
\text { en UF/ha }\end{array}$} & $\begin{array}{l}\text { Pourcentages } \\
\text { respectifs/ha } \\
\text { géographlque }\end{array}$ & $\begin{array}{r}\text { Productivi } \\
\text { en UF/ha ge } \\
\text { de bas }\end{array}$ & $\begin{array}{l}\text { moyenne } \\
\text { raphique } \\
\text { Eond }\end{array}$ \\
\hline \multirow{4}{*}{ Pâturage } & \multirow[b]{2}{*}{ Prairie hum de } & Annuelle & $\begin{array}{c}\text { Saison } \\
\text { des pluies }\end{array}$ & $\begin{array}{l}\text { Saison } \\
\text { sèche }\end{array}$ & \multirow[b]{2}{*}{$50 p .100$} & Annue11e & $\begin{array}{l}\text { Saison } \\
\text { sêche }\end{array}$ \\
\hline & & 4065 & 2930 & 1135 & & 2030 & 565 \\
\hline & \multirow[t]{2}{*}{ Colluvions } & \multirow[t]{2}{*}{2155} & \multirow[t]{2}{*}{1920} & \multirow[t]{2}{*}{235} & $30 p \cdot 100$ & 646 & 70 \\
\hline & & & & & Total & 2676 & 635 \\
\hline \multirow{3}{*}{$\begin{array}{l}\text { Pâturage } \\
\text { rationnê }\end{array}$} & Prairie humide & 3270 & 1170 & 2100 & $50 \mathrm{p} \cdot 100$ & 1635 & 1050 \\
\hline & \multirow[t]{2}{*}{ Colluvions } & \multirow[t]{2}{*}{1610} & \multirow[t]{2}{*}{1170} & \multirow[t]{2}{*}{440} & $30 \mathrm{p} .100$ & 480 & 130 \\
\hline & & & & & Total & 2115 & 1180 \\
\hline
\end{tabular}


En pâturage rationné, la productivité annuelle moyenne se maintient à environ $6 \mathrm{t} / \mathrm{ha}$ de M.S., tandis qu'en exploitation continue, elle descend à $5 \mathrm{t} / \mathrm{ha}$ la première année. Cette baisse est essentiellement due au faible développement de Hyparrhenia rufa.

Par contre, il n'y a aucune différence sensible entre les productivités en saison sèche obtenues avec les deux modes d'exploitation parce que les terres sablonneuses et perméables sont sèches et le facteur déterminant est l'eau et non le rythme des coupes.

\section{DISCUSSION DES RESULTATS}

\section{A. CONSEQUENCES PRATIQUES}

\section{Pâturages de saison des pluies}

a) Moyen-Ouest

\section{Fanage et réserve de foins}

Le rendement des regains n'est pas suffisant pour justifier une deuxième coupe. Le prix de revient du foin s'abaisse lorsque les rendements/ha s'élèvent. Il est préférable de faire une seule coupe à la floraison des graminées, et il y a intérêt à se baser sur la floraison de la plus productrice, c'est-à-dire Hyparrhenia rufa (avril).

D'autre part, étant donné que les réserves racinaires sont peu importantes (regains médiocres) on ne risque pas, avec une seule coupe en fin de végétation, d'épuiser les souches comme le feraient deux coupes successives.

\section{Pâturage}

Si l'on exploite en faisant pâturer, ce qui est le cas le plus fréquent, il faut s'efforcer de maintenir le bétail sur les pâturages des terres hautes en saison des pluies :

- puisqu'il n'y a pas de regains, le pâturage sera au repos jusqu'à la saison des pluies suivante et on ne risque pas de dégrader la végétation;

- on limite les feux courants sur les parcelles exploitées (pas de combustible);

- on exploite au maximum les potentialités de ces pâturages;

- on réserve les terres basses pour la saison sèche;
- en concentrant le bétail sur une partie seulement des surfaces disponibles dans la rotation, on accroît le recouvrement et la quantité des matières végétales sur les zones en défens, ce qui permettra d'introduire le feu à contre-saison l'année suivante sur celles-ci (6).

\section{b) Ouest}

Le même raisonnement appliqué à l'Ouest montre que, dans cette zone, on a au contraire intérêt à exploiter le pâturage naturel en deux fois.

En station pendant la saison des pluies, il est parfois impossible de pénétrer dans les parcelles avec des engins mécaniques, mais on peut faire pâturer lorsque l'herbe atteint $50 \mathrm{~cm}$ de hauteur.

On retarde ainsi les cycles végétatifs, et on peut faire un foin moins ligneux (regains) après la fin des pluies avec des rendements qui, bien qu'inférieurs à ceux donnés par une coupe unique, justifient une exploitation mécanisée. Une solution serait de faire de l'ensilage avec la première coupe et du foin avec la deuxième sur les parcelles où les engins peuvent pénétrer en février.

En élevage extensif, si l'on ne fait pas de foins, on a intérêt à déplacer le bétail au cours de la même saison des pluies pour permettre aux regains de donner un pâturage suffisant (rendement en matière sèche). Comme dans le Moyen-Ouest, une mise en défens partielle permet d'introduire le feu à contre saison, l'année suivante.

Il faut remarquer que la digestibilité du fourrage produit au cours des deuxièmes cycles (regains) risque d'être plus élevée que si l'on ne fait qu'une coupe, la teneur en lignine et hemicellulose étant moins élevée.

\section{Pâturages de saison sèche (bas-fond)}

a) Moyen-Ouest

Dans le Moyen-Ouest, il est rationnel d'exploiter essentiellement les pâturages de plateau en saison des pluies et de réserver les bas-fonds pour la saison sèche. Il n'est pas souhaitable de les laisser en défens, sinon on provoque une lignification des tiges et une augmentation considérable du pourcentage de refus (25 p. 100). Les résultats de l'expérimentation montrent qu'il est préférable d'effectuer un 
pâturage en mars pour que le bas-fond puisse être exploité intensivement à partir du mois de juin (début de la saison sèche).

Les résultats chiffrés en Unités Fourragères donnent (tableau II) pour un hectare géographique de bas-fond une production en saison sèche de :

- Exploitation continue: $635 \mathrm{UF}$.

- Exploitation rationnée : 1180 UF.

Dans les conclusions des travaux précédents (3) nous indiquions que la charge à l'hectare pouvait être évaluée à $1,5 / 1,8$ tềte UBT par ha géographique de bas-fond. Avec une exploitation intensifiée en saison sèche, la charge peut être augmentée jusqu'au maximum de 2,6 tête/ha si les superficies de pâturages de terres hautes sont suffisantes.

Lorsque l'on considère une période limitée de pâture, la charge peut être très élevée : cette notion est très souvent confirmée en milieu tropical. Par exemple, en saison des pluies, à cause de la mise en exploitation pár rotation d'un tiers des surfaces, la charge momentanée est 3 fois supérieure à la charge annuelle.

\section{b) Ouest}

Malgré l'assèchement des bas-fonds, des repousses sont pâturables en saison sèche. Contrairement à ce que l'on observe dans le MoyenOuest, le mode rationné ne donne pas un accroissement des rendements en saison sèche et d'autre part, l'exploitation continue des basfonds réduit la productivité globale annuelle.

Dans la mesure où cela est possible, il faut réserver les bas-fonds pour la saison sèche, afin de ne pas dégrader la végétation. On a observé que le pâturage en saison des pluies pouvait provoquer l'arrachement des touffes d'Hyparrhenia rufa et que le piétinement excessif détruisait en partie les germinations. Afin d'éviter une lignification excessive des chaumes, on peut faire pâturer légèrement le bétail pendant le mois d'avril.

Les feux de contre-saison donnent de bons résultats sur de tels pâturages, si l'on respecte un temps de repos de 2 mois.

La charge à l'hectare limitante en saison sèche reste inchangée; elle est de l'ordre de 1,6 ha de bas-fond par tête si l'on applique le même raisonnement que pour le Moyen-Ouest.

Si les superficies de bas-fond sont insuffisantes, il devient indispensable de supplémenter le bétail.

\section{B. RELATIONS CLIMAT/SOLS/VEGETATION}

Si nous considérons l'association végétale à Hyparrhenia rufa dominant dans les deux régions étudiées, nous remarquons qu'elle est située dans des zones différentes de la catena.

Dans le Moyen-Ouest elle recouvre les plateaux, alors que dans l'Ouest elle est localisée sur les bas de pentes. Nous savons que les exigences écologiques d'une association sont beaucoup plus strictes que celles d'une espèce, aussi il n'est pas sans intérêt d'établir la comparaison entre les deux milieux.

La comparaison de ces différents facteurs écologiques permet d'expliquer la présence de l'association à Hyparrhenia sur des milieux différents.

TABLEAU

\begin{tabular}{|c|c|c|}
\hline Caractéristiques & Moyen-Quest & Ouest \\
\hline \multicolumn{3}{|l|}{ Climat } \\
\hline $\begin{array}{l}\text { Pluviométrie } \\
\text { Température moyenne annuelle } \\
\text { Nombre de jours de pluies }\end{array}$ & $\begin{array}{r}1.600 \\
21 \\
120\end{array}$ & $\begin{array}{r}1.200 \\
27 \\
80\end{array}$ \\
\hline Sols & $\begin{array}{l}\text { Ferralitiques à horizon compact } \\
\text { moyennement désaturés }\end{array}$ & $\begin{array}{l}\text { Ferrugineux, sableux bien } \\
\text { pourvus en matière organique } \\
\text { et minéraux }\end{array}$ \\
\hline \multicolumn{3}{|l|}{ Eau } \\
\hline $\begin{array}{l}\text { Capacité de retention de l'eau } \\
\text { Répartition locale des pluies }\end{array}$ & $\begin{array}{l}\text { Elevée dans les argiles } \\
\text { Drainage vertical vers } \\
\text { les bas-fonds }\end{array}$ & $\begin{array}{l}\text { Faible dans les sables } \\
\text { Drainage latéral et accumulation } \\
\text { en bas de pentes }\end{array}$ \\
\hline
\end{tabular}


L'insuffisance des pluies dans l'Ouest est compensée par l'accumulation locale des eaux de drainage, des forces de rétention peu élevées dans les sables, une teneur en matière organique élevée qui accumule plus d'eau et qui constitue une réserve d'éléments minéraux utilisables par les plantes après minéralisation (1).

Lorsque les exigences d'une association végétale sont connues, on peut affirmer que, si le climat devient plus rigoureux, elle sera localisée sur des sols plus riches.

\section{CONCLUSIONS}

Le rythme des coupes peut avoir une influence sur la productivité des pâturages. Dans le Moyen-Ouest, les pâturages des terres hautes ont une productivité qui n'est pas influencée par le nombre des coupes en saison des pluies, tandis que dans les bas-fonds on peut accroître les rendements des coupes en saison sèche si l'on allonge les temps de repos en saison des pluies.
Dans l'Ouest où les bas-fonds se dessèchent progressivement pendant la saison sèche mais où des conditions écologiques particulières sont favorables à la pousse de l'herbe en saison des pluies, l'augmentation du nombre des coupes accroît la productivité des pâturages des terres hautes, mais les rendements en bas-fonds ne sont pas influencés par les variations des durées des temps de repos.

Ces résultats peuvent être interprétés si l'on considère l'ensemble des facteurs écologiques.

Dans les bas-fonds du Moyen-Ouest, l'eau est en quantité suffisante pendant toute la saison sèche pour permettre une croissance continue de l'herbe, alors que dans l'Ouest l'évaporation et l'infiltration assèchent les sols.

Depuis toujours, ces particularités connues empiriquement des éleveurs ont été à la base des mouvements de troupeaux qui se déplacent vers les zones de transition du Moyen-Ouest en saison sèche, fait le plus marquant de l'économie de l'élevage à Madagascar.

\section{SUMMARY}

Inlfuence of the method of management on natural grassland productivity in Madagascar

The method of management (dates and number of cuttings) influences differently overall and seasonal productivity according to grassland type (wet or dry area) and climate.

Experiments make it possible to define more exactly relationships between climate, soils and vegetation and recommand a management method depending on the crop considered and the area concerned.

\section{RESUMEN}

Influencia del modo de explotación sobre la productividad de los pastos naturales de Madagascar

El modo de explotación (fechas y número de siegas) influye sobre la productividad global y estacional diferentemente según el tıpo de pasto (zona húmeda o seca) y el clima.

La experimentación permitió precisar las relaciones entre el clima, los suelos y la vegetación y preconizar un modo de explotación en función a la vez de la especulación considerada y de la zona interesada.

\section{BIBLIOGRAPHIE}

1. BOURGEAT (F.). Etude de la basse vallée du Kamoro. Tananarive, ORSTOM, 1964.

2. DELAPORTE (J.). Contribution à l'étude du comportement des bovins en élevage extensif à Madagascar. Thèse, Doct. vét. Lyon, 1971.

3. GRANIER (P.), LAHORE (J.), DUBOIS (P.). Etude du pâturage naturel à Madagascar. Productivité, conséquences pratiques. Rev. Elev. Méd. vét. Pays trop., 1968, 21 (2): 203-217.

4. GRANIER (P.). Productivité du pâturage naturel de bas-fond dans le Moyen-Ouest de Madagascar. Terre Malgache, 1970, (8): 167-178.

5. GRANIER (P.). Influence du mode d'exploitation du pâturage sur les rendements en matière sèche. Rapport I.E.M.V.T., juin 1970.

6. GRANIER (P.), GILIBERT (J.). Contribution à l'étude de l'exploitation par rotation dés pâturages de savane soudanienne. Rev. Elev. Méd. vét. Pays trop., 1974, 27 (2) : 223-233.

7. LEMEE. Précis de Biogéographie. Paris, Masson. 